Kansas State University Libraries

New Prairie Press

\title{
ANALYSIS OF GENOTYPE X ENVIRONMENT INTERACTION BY GRAPHICAL TECHNIQUES
}

George C.J. Fernandez

Follow this and additional works at: https://newprairiepress.org/agstatconference

Part of the Agriculture Commons, and the Applied Statistics Commons

\section{(c) (1) $\Theta(9$}

This work is licensed under a Creative Commons Attribution-Noncommercial-No Derivative Works 4.0 License.

\section{Recommended Citation}

Fernandez, George C.J. (1991). "ANALYSIS OF GENOTYPE X ENVIRONMENT INTERACTION BY

GRAPHICAL TECHNIQUES," Conference on Applied Statistics in Agriculture. https://doi.org/10.4148/

2475-7772.1424

This is brought to you for free and open access by the Conferences at New Prairie Press. It has been accepted for inclusion in Conference on Applied Statistics in Agriculture by an authorized administrator of New Prairie Press. For more information, please contact cads@k-state.edu. 


\title{
ANALYSIS OF GENOTYPE X ENVIRONMENT INTERACTION BY GRAPHICAL TECHNIQUES
}

\author{
George C.J. Fernandez \\ Department of Agricultural Economics \\ University of Nevada- Reno \\ Nevada Reno NV 89557
}

\begin{abstract}
Genotype $x$ Environment interactions results from the changes in the magnitude of differences among genotypes (non-crossover or quantitative interactions) or changes in the relative ranking of the genotypes (crossover or qualitative interactions) in different environments. Non-crossover interactions are usually associated with variance heterogeneity and non-additivity. The analysis of variance combined with joint regression analysis failed to differentiate between the crossover and non-crossover interactions. Tedious computations are necessary in comparisons of all possible pairs of genotypes in all possible pairs of environments in the crossover detection tests. Therefore, differentiating the non-crossover interaction caused by variance heterogeneity and non-additivity from crossover interaction by simple but effective methods such as exploratory data analysis should be carried out before assessing the stability in GEI studies. The effectiveness of the four graphical methods i) variance heterogeneity diagnostic plot (Box et al. 1978), ii) transformable non-additivity diagnostic plot, (Box et al. 1978) iii) Emerson and Hoaglin's (1983) nonadditivity diagnostic plot, and iii) Gabriel's bi-plot (1971) in detecting non-crossover interactions resulting from variance heterogeneity and nonadditivity are presented in this paper. Baker's (1990) three simulated and the spring wheat data sets were used to evaluate the effectiveness of these four graphical techniques.
\end{abstract}

Key words: crossover and non-crossover interactions; variance heterogeneity; transformable non-additivity diagnostic plot; bi-plot; exploratory data analysis.

\section{Introductions}

Genotype x Environment Interaction (GEI) is an important consideration in multi-environmental plant breeding trials, when the test genotypes exhibit inconsistent relative performance over environments. 
Significant GEI results from the changes in the magnitude of differences among genotypes or changes in the relative ranking of the genotypes in different environments. The GEI when present reduces the progress from selection in any one environment.

A qualitative or crossover interaction arises when the ranks of the true genotype mean vary among the environments while the quantitative or non-crossover interaction involves the variation in the magnitude of genotype means among environments (Peto, 1982; Gail and Simon, 1985). Haldane (1946) and Gregorius and Namkoong (1986) claimed that only the crossover interaction is practically important. Non-crossover interactions are usually associated with variance heterogeneity and nonadditivity of the GE data matrix. Thus, differentiating between the crossover and non-crossover design is important in decision making relating to plant breeding strategies, since the presence of crossover interaction emphasizes the need for breeding programs for specific adaptation to certain environments.

Several statistical methods are proposed to assess the GEI or the environmental sensitivity of genotypes tested under a wide range of environments (Fernandez, 1991). The most popular methods have used analysis of variance combined with joint regression analysis to determine the environmental sensitivity of the genotypes (Finlay and Wilkinson, 1963; Eberhart and Russell, 1966; Fernandez et al. 1989). When the genotype mean in an environment is regressed on an environmental index (environment mean - grand mean), the regression coefficient and a function of squared deviation from the regression would provide estimates of environmental sensitivity statistics. With this model, the sums of squares due to environments and genotype $\mathrm{x}$ environments are partitioned into environment-linear, GxE-linear, and deviation from the regression model. Baker (1990) used the conventional joint regression analysis on three simulated data sets and concluded that this method failed to differentiate between the crossover and non-crossover interactions.

For detecting crossover interaction, Baker (1990) used the tests developed by Azzalini and Cox (1984) and Gail and Simon (1985). Tedious computations involving comparisons of all possible pairs of genotypes in all possible pairs of environments are required in these tests. For example, 3620 possible quadruple comparisons are necessary for a $9 \times 10$ GE data matrix (Baker, 1990). Therefore, differentiating the noncrossover interaction caused by variance heterogeneity and non-additivity from crossover interaction by simple but effective methods such as exploratory data analysis 
should be carried out before assessing the stability in GEI studies.

Exploratory data analysis is the "manipulation, summarization, and display of the data to make them more comprehensible to human minds; thus uncovering the underlying structure in the data and detecting important departure from this structure" (Andrews, 1978). Examining a plot of predicted values and the residuals is a widely used exploratory data analysis to detect the violation of assumptions in ANOVA and regression models (Fernandez, 1992). The feasibility of using some graphical techniques to detect the noncrossover interactions should be evaluated as alternative to the complex crossover detection tests.

The objectives of this study were to investigate the effectiveness of the four graphical methods $i$ ) variance heterogeneity diagnostic plot (Box et al. 1978), ii) transformable non-additivity diagnostic plot, (Box et al. 1978) iii) Emerson and Hoaglin's (1983) non-additivity diagnostic plot, and iv) Gabriel's bi-plot (1971) in detecting non-crossover interactions resulting from variance heterogeneity and non-additivity. Baker's (1990) three simulated and the spring wheat data sets were used to evaluate the effectiveness of these four graphical techniques.

\section{Baker's (1990) simulated data sets:}

\subsection{Random effects model:}

The statistical model, Baker (1990) used for generating simulated data was:

$Y_{i j}=\mu+G_{i}+E_{j}+G E_{i j}$

where $G_{i}, E_{j}$, and $G E_{i j}$ represent random genotype, environmental, and GEI effects respectively. The data comprise of 8 genotypes evaluated in 10 environments (table 1). In this model, the changes in genotype rank result from differences in genotype response to unpredictable factors in the environment. This model should show no relationship between the GEI effects and the environmental deviation. However, joint regression analysis produced regression coefficients that vary from - .2 to .2 particularly for this small simulated data with eight genotypes. The range in regression coefficient decreases as the number of genotypes increases. In this simulated data, about $10 \%$ of the GEI variance was attributed to the heterogeneity of regression coefficients (Baker 1990). 


\subsection{Multiplicative model:}

Baker (1990) used the following model to generate a simulated data with multiplicative effects:

$$
Y_{i j}=\mu+\left(G_{i}-G_{\min }\right)\left(E_{j}-E_{\min }\right)
$$

Where, $Y_{i j}$ is the function of the product of random normal deviate $G_{i}$ and $E_{j}$. This data also comprise of 8 genotypes evaluated in 10 environments (table 1 ). The important feature of this model is that there was no crossover interaction. This type of non-crossover interaction involves heterogeneity of variance, nonadditivity, and high correlation among environments. significant regression coefficients, insignificant mean square deviation from the joint regression analysis, and a positive correlation between genotype mean and the regression coefficients were characteristic of this data.

\subsection{Yield-disease model:}

The simulated data for this model were generated by the following statistical model (Baker 1990)

$Y_{\mathrm{ij}}=\mu+G_{i}+E_{j}-B_{i}\left(E_{j}-E_{\min }\right)$

where $B_{i}$ is 0 for resistant genotypes and 0.3 for susceptible genotypes. The disease incidence is assumed to be greater under environmental condition that favors higher yield. This data also comprise of 8 genotypes evaluated in 10 environments (table 1). As in the multiplicative model, the interaction was reflecting a non-additive model and there were no deviations from the joint regression analysis. The yield disease model differs from the multiplicative model in that crossover interactions occurred and differed from the random effects model in that the crossover interactions were related to specific causes, the degree of disease resistance.

\subsection{Spring wheat yield data (Baker 1990):}

This data comprise of nine genotypes grown in 10 environments (table 1). Significant crossover interaction was observed as in yield disease model due to differential disease resistance potentials. 


\section{Graphical technigues to detect non-crossover interaction:}

3.1 Variance heterogeneity diagnostic plot (Box et al. 1978):

A plot between log of environmental standard deviation $\left(S_{. j}\right)$ and the log of environmental mean $\left(\bar{Y}_{. j}\right)$ allows to judge the extent of variance heterogeneity and suggests which power transformation if any would help to remove it. A scatter plot with no consistent trend indicates the absence of variance heterogeneity and a linear trend suggests the presence of variance heterogeneity. If the regression is not significant when $\log \left(S_{. j}\right)$ regressed on $\log \left(\bar{Y}_{. j}\right)$, data transformation is usually not necessary. A significant regression $(P<0.05)$ indicates the data should be transformed and the regression coefficient is estimated. The power $(\lambda)$ of the transformation can be estimated by subtracting the regression coefficient $(\beta)$ from 1 . The value of the power, $(\lambda)$ indicates the appropriate transformation. For example, if $\beta$ approximately equals 2 , then $\lambda=1-\beta$ $=-1$. Thus, the appropriate transformation would be reciprocals.

3.2 The transformable additivity diagnostic plot (Box et al. 1978):

In a two-way data structure, a value of the response is observed for each combination of genotype and environment. Where:

$Y_{i j}=\mu+G_{i}+E_{j}+G E_{i j}$.

First an additive model is fitted and the $\mu_{i} G_{i}$, and $E_{j}$ are estimated. The transformable additivity diagnostic plot (Box et al. 1978) plot illustrates the relationship between the interaction component, $G E_{i j}$ and $\bar{Y}_{\mathrm{ij}}$, where

$\bar{Y}_{i j}=\mu+G_{i}+E_{j}$

$G E_{i j}=Y_{i j}-\bar{Y}_{i j}$

A scatter plot with no consistent trend indicates the absence of transformable non-additivity and a curvilinear plot suggests the existence of noncrossover interaction resulting from transformable nonadditivity. 
3.3 Emerson and Hoaglin's (1983) non-additivity

diagnostic plot:

This plot allows the extent of any non-additivity to be judged and suggests which power transformation if any would help to remove it. The diagnostic plot

illustrates the relationship between the interaction component, $G E_{i j}$ and the comparison value $\left(C V_{i j}\right)$, where:

$C V_{\mathrm{ij}}=\left(\begin{array}{lll}G_{\mathrm{i}} & \mathrm{x} & E_{\mathrm{j}}\end{array}\right) / \mu$

A scatter plot with no consistent trend indicates that the data do not depart systematically from an additive model. Alternatively, if we see a linear plot, the slope of this diagnostic plot guides us to a transformation that should help to remove the nonadditivity. If the slope is $\beta$, the power near (1- $\beta$ ) provide a useful power transformation.

\subsection{Gabriel's biplot (1971):}

The $G E_{i j}$ component is partitioned into a sum of multiplicative terms using principal component analysis:

$$
Y_{i j}=\mu+G_{i}+E_{j}+\sum a_{i l} b_{j l}+\varepsilon_{i j}
$$

Let $\lambda_{u}$ is the $u^{\text {th }}$ eigenvalue and $b_{1 u}, b_{2 u}$. . Is the $u^{\text {th }}$ eigenvector. When scaled so that $\sum_{j} b_{j u}^{2}=1$, the $b_{1 u}$, $\mathrm{b}_{2 u}, \ldots$ represents the envirommental 10 adings for the $u^{\text {th }}$ principal component while $a_{1 u}, a_{2 u}$. . represent the genotype scores on that principal component axis, and $\lambda_{u}=\sum_{i} a^{2}$ is is the contribution to the GEI sums of squares arising from the $u^{\text {th }}$ component (Kempton, 1984). Thus, the biplot display of principal component analysis where two dimensional approximation to a two way table (rows $x$ column) can be obtained from the first two principal components. For a table consisting of GEI components, the genotypes (row points) and environments (vector coordinates) can be plotted in the same graph (the biplot).

In all four data sets, number of environments (columns) were greater than the number of genotypes. To obtain unique principal components, the number of row points (observations) should be greater than the columns (variables). To satisfy this requirement and to be consistent with all four data analyses, certain environments were not included in all four graphical analyses (table 1 ). 


\section{Results and Discussions}

The Box et al. (1978) variance heterogeneity diagnostic plots (Fig.1) revealed the presence of significant heterogeneous variances among environments in the multiplicative and yield disease models. A scatter plot with no consistent trend in the random effects model confirmed variance homogeneity. In addition, 1-slope of the linear regression model between the $\log S_{j}$ and $Y_{j}$ can be useful in deciding the suitable power transformation that can be used to remove the variance heterogeneity. The suggested power transformations that can remove the variance heterogeneity were $\mathrm{Y}^{-2.92}, \mathrm{Y}^{-2.66}$, and $\mathrm{Y}^{0}$ for the multiplicative, yield disease, and the spring wheat data respectively. However, the suggested power transformations based on the slope were not appropriate in the context of the data for the multiplicative and the yield disease models.

A curvilinear plot in the transformable additivity diagnostic plots (Box et al.1978) (Fig.2) confirmed the presence of non-crossover interaction in addition to variance heterogeneity in the multiplicative model. scatter plots were observed in other three models confirmed the presence of crossover interactions.

Scatter plots were observed in Emerson and Hoaglin's non-additivity plots for the random effects and spring wheat data (Fig. 3) confirmed the absence of nonadditivity in these models. A highly significant linear relationship in the multiplicative model confirmed the presence of non-additivity or the non-crossover interaction. The recommended power transformation to remove non-additivity based on the slope was -3.7 . However, this is not an appropriate power transformation within the context of the data. In the yield disease model, a significant linear trend was observed. This could be attributed to the variance heterogeneity of the yield disease data. Thus, Emerson and Hoaglin's nonadditivity plot failed to separate non-additivity and variance heterogeneity when they are present together.

The biplot displays points for genotypes and vector coordinates for environments on the same plot so that the expected response of a genotype in a particular environment may be derived from visual inspection of their relative positions on the biplot. The biplot will display a major portion the variation of the two-way GEI data matrix. The relative length of the vector line will represent the variance of the environments and their angles represent their correlations. The ranking of genotypes in a particular environment will be achieved by ordering the projected points. 
In the multiplicative model, the presence of transformable non-additivity was revealed by the highly significant correlations (0.999) among the high yielaing and the low yielding environments. About 99.9\% variation in the data could be explained by the first dimension in the biplot display which separated the low yielding environments (Envi-Env4) from the high yielding environments (Env5-Env10) (Fig. 4).

In the yield disease model, the first dimension which explains $88 \%$ of the variation separated the low yielding environments (Env1-Env4) from the high yielding environments (Env5-Env10). The nature of the crossover interactions in the yield disease model was revealed by the pattern of grouping of similar environments and genotypes and the separation of dissimilar environments by the relative positions of the genotypes in the biplot. The relatively higher performance of disease resistant genotypes, $B, D$, and $E$ in high yielding environments where the disease pressure is expected to be higher is an indication of crossover interaction.

In the spring wheat data, the first dimension which explains $76 \%$ of the variation separated the high yielding environments (Env3, Env6, and Env10) from the low yielding environments. The nature of the crossover interactions in this data was revealed by the position of interacting genotypes and environments in the biplot. Relatively higher yields of genotypes $C$ and $G$ in the high yielding environments and relatively lower yields of genotypes $D$ and $I$ in the low yielding environments clearly indicate the presence of crossover interaction in this data.

\section{5. summary}

The variance heterogeneity diagnostic plot effectively detects the heterogeneity of variance. The transformable non-additivity could be separated from crossover interaction by the transformable non-additivity diagnostic plots (Box et al.1978) and the biplots. Noncrossover interaction attributed to both variance heterogeneity can be detected by the presence of significant linear plot in the Emerson and Hoaglin's nonadditivity plots. In addition, the biplot technique is useful in showing inter-unit distances among the genotypes and environments and thus facilitate the clustering of genotypes as well as display variances and correlations among the environments. Thus, these exploratory graphical techniques are valuable tools in the GEI studies before estimating and interpreting the traditional stability statistics. 


\section{References}

Andrews, D. 1978. Exploratory data analysis. In: International Encyclopedia of statistics. PP. 97-107. Krypton W. H. (edS.). N.Y. Free Press.

Azzalini, A. and D.R. Cox. 1984. Two new tests associated with analysis of variance. J. R. Stat. Soc. B. 46:335-343.

Baker, R.J. 1990. Crossover genotype-environmental interaction in spring wheat. In genotype by environment interaction and plant breeding. $M$. S. Kang (ed.) Louisiana Agricultural Experiment station. ISU. Baton Rouge. Box, G.E.P., W.G. Hunter, and J.S.

Hunter. 1978. Statistics for Experimenters. John wiley \& Sons. N.Y.

Eberhart, S.A, and W.A. Russell. 1963. Stability parameters for comparing varieties. Crop sci. 6: 36-
40 .

Emerson J.D. and D.C. Hoaglin.1983. Analysis of two-way tables by medians. In: Understanding Robust and exploratory data analysis. D.C. Hoaglin, F. Mosteller, and J.H. Tukey. (eds). John Wiley \& Sons. Inc. N.Y.

Fernandez, G.C.J., H.K. Chen and J.C. Miller, Jr. 1989. Adaptation and environmental sensitivity of mungbean genotypes evaluated in the International Mungbean Nursery. Euphytica 41:253-261.

Fernandez, G.C.J. 1991. Analysis of genotype $x$ environment interaction by stability estimates. HortSci. 26:947-950.

Fernandez, G.C.J. 1992. Residual analysis and transformations - Important tools in statistical analysis. Hortsci. $27:$ (in press).

Finlay, K.W. and G.N. Wilkinson, 1963. The analysis of adaptation in plant breeding programs. Aust. J. Agric. Sci. $14742-757$.

Gabriel, K.R. 1971. Biplot display of multivariate means with application to principal component analysis. Biometrika 58:453-467.

Gail, $M$. and R. Simon. 1965. Testing for qualitative interactions between treatment effects and patient subsets. Biometrics 41:361-372.

Gregorius, H.R. and G. Namkoong. 1986. Joint analysis of genotype $x$ environmental effects. Theor. Appl. Genet. $72: 413-422$.

Haldane, J.B.S. 1946. The interaction of nature and nurture. Ann. Eugenics. 13:197-205.

Kempton, R.A. 1984 . The use of biplots in interpreting variety by environment interactions. J. Agric. Sci. $103: 123-135$.

Peto, R. 1982. statistical aspects of cancer trials. PP:867-871. In: E.E. Halnan. (ed.) Treatment of cancer. Chapman and hall. London. U.K. 
Table 1. Baker's (1990) data sets.

1. Random effects model

\begin{tabular}{|c|c|c|c|c|c|c|c|c|c|c|}
\hline Gen. & \pm 1 & $\mathrm{E} 2$ & $E 3^{z}$ & $E 4$ & E5 & $E 6^{z}$ & E7 & $\mathbb{E} 8^{z}$ & E9 & E10 \\
\hline A & 170 & 173 & 180 & 184 & 202 & 193 & 171 & 218 & 207 & 35 \\
\hline B & 154 & 190 & 172 & 191 & 187 & 192 & 192 & 209 & 211 & 1 \\
\hline C & 185 & 183 & 181 & 214 & 197 & 211 & 203 & 213 & 214 & 39 \\
\hline D & 177 & 195 & 194 & 208 & 203 & 192 & 216 & 213 & 201 & \\
\hline$E$ & 190 & 204 & 210 & 193 & 199 & 211 & 204 & 239 & 235 & 2 \\
\hline T & 192 & 176 & 206 & 218 & 213 & 213 & 195 & 221 & 244 & \\
\hline & 170 & 204 & 196 & 214 & 221 & 213 & 214 & 240 & 243 & \\
\hline & 202 & 228 & 209 & 218 & 235 & 239 & 246 & 254 & 263 & \\
\hline
\end{tabular}

\section{Multiplicative model}

\begin{tabular}{|c|c|c|c|c|c|c|c|c|c|c|}
\hline Gen. & E1 & $\mathbf{E} 2^{z}$ & 23 & $\mathbb{E} \mathbf{S}^{z}$ & E5 & $\mathbb{E} 6$ & E7 & E8 & $E g^{z}$ & E10 \\
\hline A & 200 & 200 & 200 & 200 & 200 & 200 & 200 & 200 & 200 & 200 \\
\hline T & 200 & 203 & 204 & 206 & 207 & 207 & 209 & 211 & 213 & 215 \\
\hline & 200 & 215 & 227 & 236 & 241 & 244 & 256 & 268 & 279 & 291 \\
\hline & 200 & 216 & 229 & 239 & 245 & 248 & 261 & 273 & 285 & 299 \\
\hline & 200 & 220 & 236 & 248 & 255 & 258 & 275 & 290 & 304 & 321 \\
\hline & 200 & 232 & 257 & 278 & 289 & 294 & 321 & 345 & 368 & 395 \\
\hline & 200 & 236 & 263 & 286 & 297 & 303 & 333 & 359 & 385 & 1 \\
\hline & 200 & 236 & 264 & 287 & 299 & 305 & 335 & 362 & 388 & 1 \\
\hline
\end{tabular}

\section{Yield-disease model}

\begin{tabular}{|c|c|c|c|c|c|c|c|c|c|c|}
\hline Gen. & E1 & $\mathrm{E} 2^{z}$ & E3 & $E 4^{z}$ & E5 & E6 & E7 & 28 & $E 9^{z}$ & \\
\hline A & 163 & 168 & 169 & 170 & 177 & 177 & 179 & 181 & 188 & \\
\hline B & 164 & 174 & 175 & 176 & 191 & 192 & 195 & 200 & 214 & \\
\hline C & 172 & 177 & 177 & 178 & 186 & 186 & 188 & 190 & 197 & 9 \\
\hline D & 176 & 186 & 187 & 188 & 203 & 204 & 207 & 212 & 226 & \\
\hline$E$ & 177 & 186 & 187 & 189 & 204 & 204 & 208 & 212 & 226 & \\
\hline$F$ & 178 & 183 & 184 & 184 & 192 & 192 & 194 & 196 & 203 & \\
\hline G & 181 & 186 & 186 & 187 & 194 & 194 & 196 & 199 & 206 & \\
\hline$H$ & 181 & 186 & 187 & 187 & 195 & 195 & 197 & 199 & 206 & \\
\hline
\end{tabular}

4. Spring wheat (9) $x$ Environment (10)-grain vield

\begin{tabular}{|c|c|c|c|c|c|c|c|c|c|c|}
\hline Gen. & $E 1$ & E2 & E3 & $\Sigma \frac{4}{2}$ & $E 5^{x}$ & $\Xi \sigma^{z}$ & E7 & 58 & $\overline{E 9}$ & EI0 \\
\hline A & 294 & 348 & 428 & 432 & 485 & 478 & 213 & 317 & 204 & 354 \\
\hline B & 325 & 404 & 421 & 452 & 548 & 448 & 240 & 345 & 226 & 355 \\
\hline C & 305 & 314 & 427 & 463 & 486 & 506 & 214 & 316 & 196 & 353 \\
\hline D & 332 & 380 & 402 & 403 & 424 & 376 & 214 & 326 & 219 & 349 \\
\hline$E$ & 284 & 290 & 361 & 396 & 418 & 386 & 194 & 273 & 189 & 320 \\
\hline$F$ & 270 & 289 & 344 & 365 & 422 & 387 & 169 & 270 & 175 & 306 \\
\hline G & 227 & 324 & 399 & 424 & 450 & 464 & 175 & 272 & 177 & 343 \\
\hline $\mathrm{H}$ & 307 & 285 & 373 & 410 & 453 & 396 & 204 & 282 & 184 & 326 \\
\hline$I$ & 260 & 314 & 409 & 316 & 351 & 364 & 176 & 291 & 183 & 328 \\
\hline
\end{tabular}

z Yield data from these environments were not analyzed. 


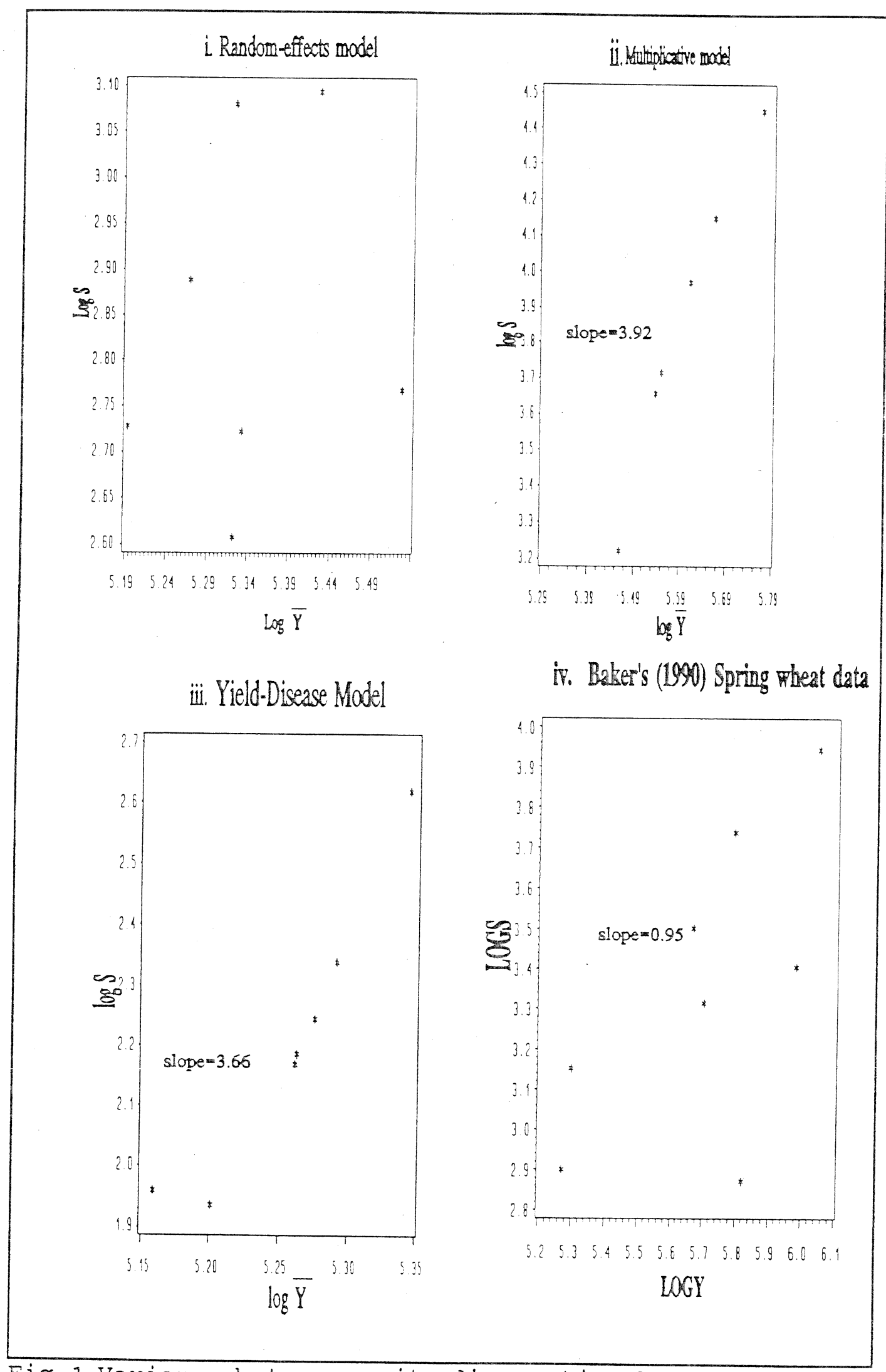

Fig. I Variance heterogeneity diagnostic plots (Box et al. 1978) 


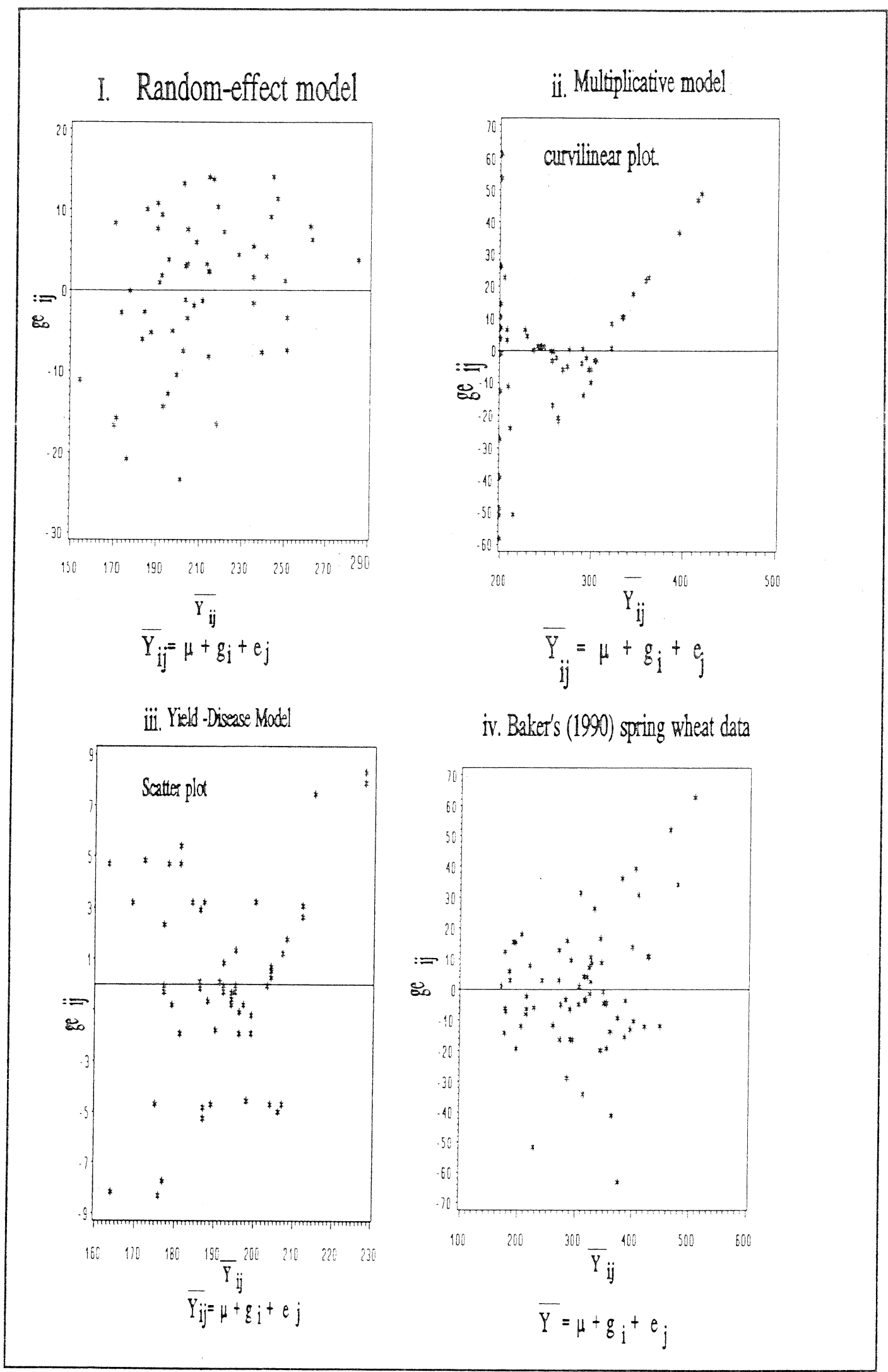

Fig.2 The transformable additivity diagnostic plots (Box et al. 1978) 


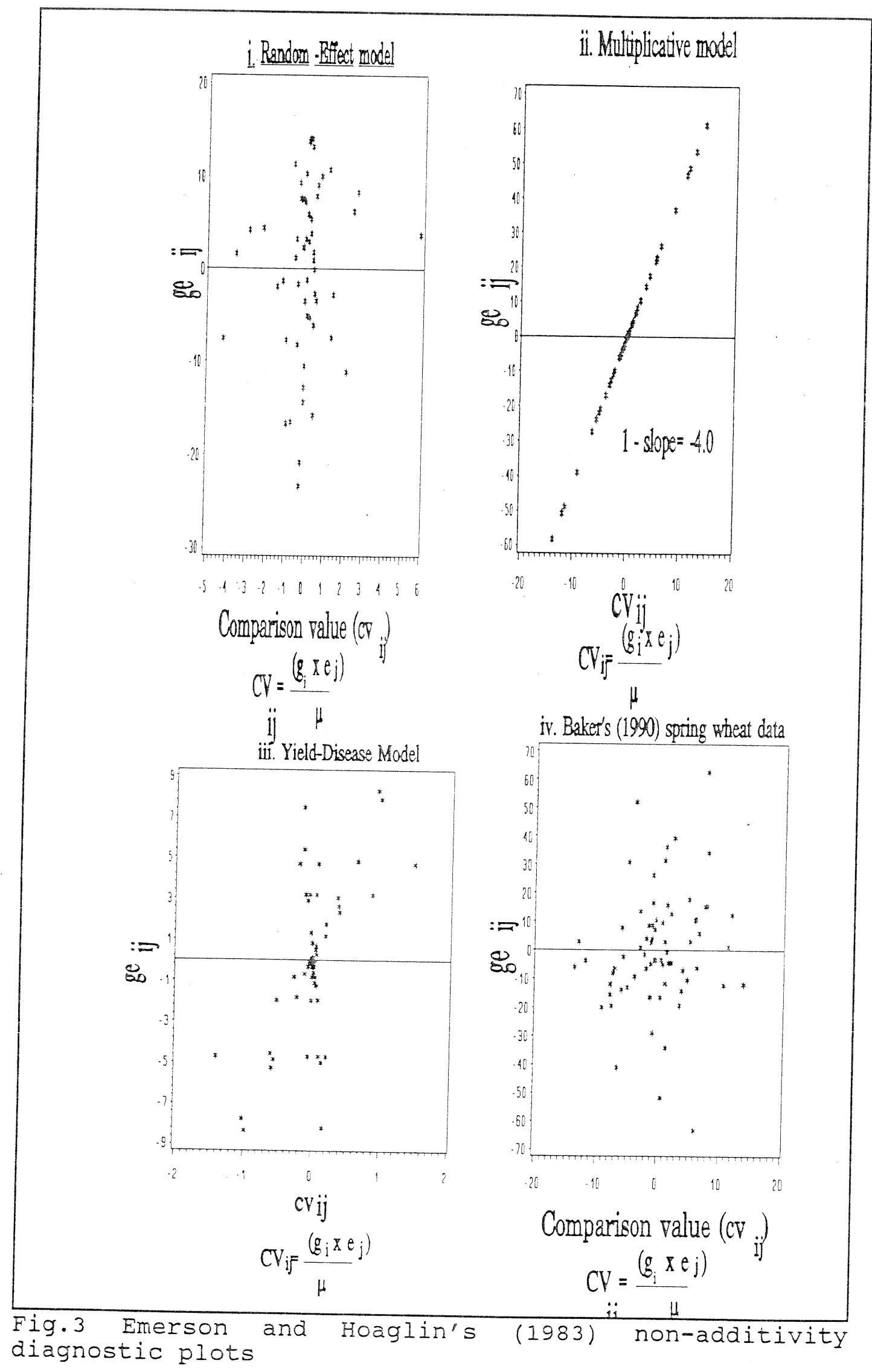




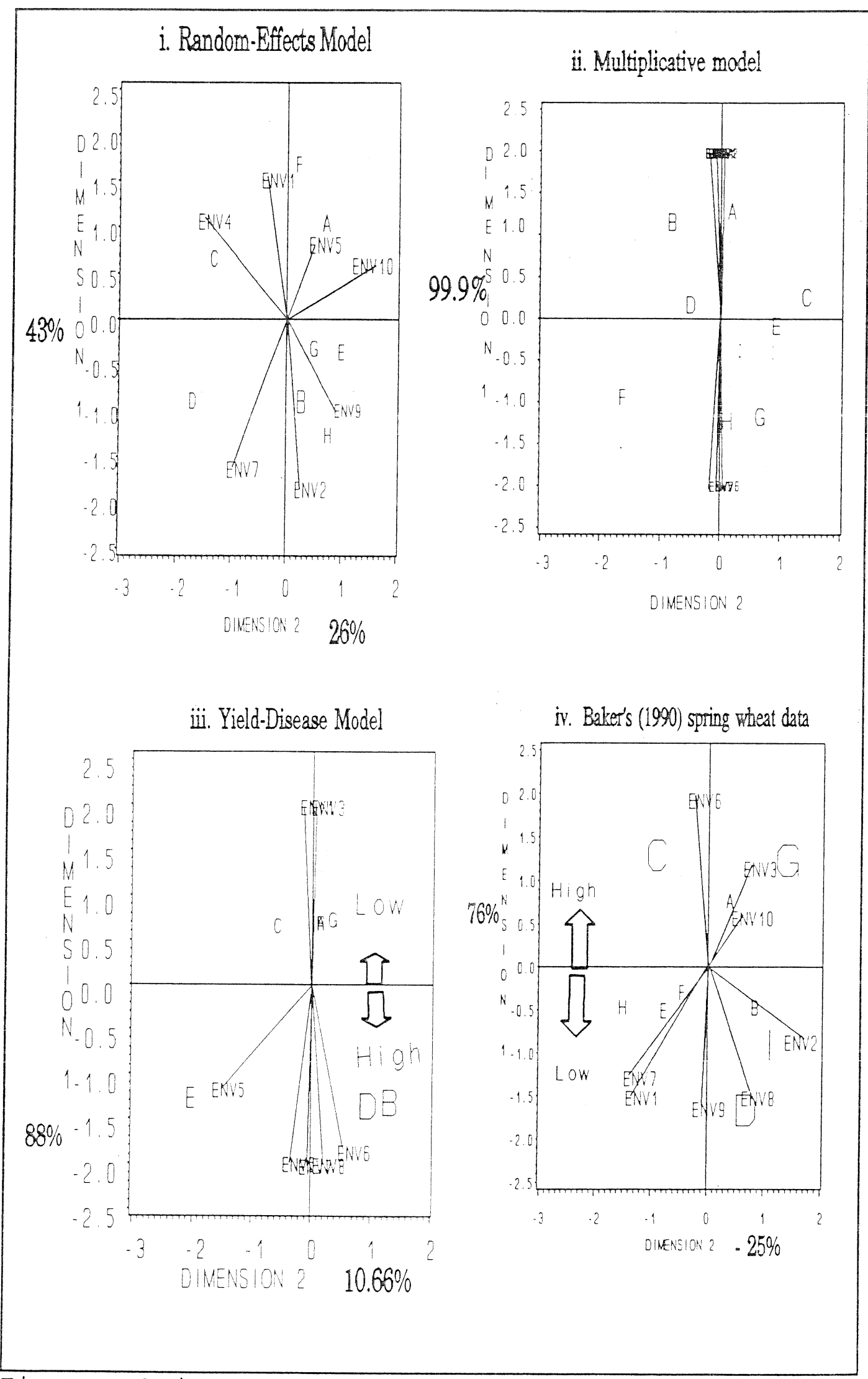

Fig.4 Gabriel's (1971) biplot representation of GEI. 